\title{
Neural interface technologies: applications of biomedical engineering to neurosurgery
}

\author{
MARK LIKER, M.D. \\ USC Department of Neurological Surgery, Keck School of Medicine, University of Southern \\ California, Los Angeles, California
}

The neurosurgical approach to diseases of the nervous system continues to evolve. As bioengineering technologies have advanced, an ally has been found to expand the role of the neurosurgeon in treating diseases not previously considered eligible for intervention. Not only are they purveyors of life-saving surgical treatments, but neurosurgeons have also embraced the application of new therapies for pain and disability, treating these as targeted disease entities in and of themselves. Consider the dramatic rise in implant technologies such as spinal pedicle screws and spinal cord stimulators to treat back pain, deep brain stimulators for functional disabilities, vagal nerve stimulators for epilepsy and depression, and peripheral nerve stimulators for pain and urinary incontinence. Sensory augmentation devices such as retinal prostheses and cochlear implant devices are in various stages of development by non-neurosurgeons and engineers. Technological developments have provided neuroscience-based clinicians with a venue to consider applications well beyond the purview of their predecessors. Many lessons can be gleaned from these and other "foreign" technologies, thereby accelerating the collaboration between the previously disparate disciplines of engineering and surgery and providing a venue for shared interests in the field of neural interface devices. In the future, leading neurosurgeons will continue to drive their craft forward by identifying the potential neurosurgical uses for emerging technology, directing engi- neering concepts toward surgical applications, and incorporating the technologies into daily practice.

In this issue, Loeb, et al., describe the BION (or bionic neuron) device and its potential for use in certain indications. This technology has many potential uses both as a stimulator and sensor, and the potential for many clinical applications yet to be investigated. Slevin, et al., present the results of an important investigation supporting the positive response that some patients with Parkinson disease experienced in earlier glial cell line-derived neurotrophic factor infusion studies, a response that was not identified in the Phase II trial. Differences in catheter type or infusion rate may explain the significant overall benefit seen in the study group. In addition, the dramatic return of the disease state to its preinfusion level after withdrawal of the new treatment reinforces the need to continue investigations of this modality. Despite advances in the field of chip design, clinicians have identified difficulty implanting cortical devices due to the strength of the pia mater. House, et al., describe a simple technique and device for such implantation. Other difficulties may be expected in patients with significant sensorimotor cortical atrophy, as described in Bakay's case report. Matsuoka, et al., delve into the design of a robotic arm that would communicate with a brain-machine interface, and Sclabassi, et al., describe and posit a set of solutions for the communications platform and power requirements of a brain-machine interface. 